\title{
Emergent Einstein Equation in $p$-adic Conformal Field Theory Tensor Networks
}

\author{
Lin Chen $\oplus^{1,2,3, \dagger}$ Xirong Liu $\oplus^{1,2,3}$ and Ling-Yan Hung $\oplus^{1,2,3,4, *}$ \\ ${ }^{1}$ State Key Laboratory of Surface Physics, Fudan University, 200433 Shanghai, China \\ ${ }^{2}$ Shanghai Qi Zhi Institute, 41st Floor, AI Tower, No. 701 Yunjin Road, Xuhui District, Shanghai 200232, China \\ ${ }^{3}$ Department of Physics and Center for Field Theory and Particle Physics, Fudan University, Shanghai 200433, China \\ ${ }^{4}$ Institute for Nanoelectronic Devices and Quantum Computing, Fudan University, 200433 Shanghai, China
}

(Received 9 March 2021; accepted 5 October 2021; published 23 November 2021)

\begin{abstract}
We take the tensor network describing explicit $p$-adic conformal field theory partition functions proposed in [L.-Y. Hung et al., J. High Energy Phys. 04 (2019) 170], and consider boundary conditions of the network describing a deformed Bruhat-Tits (BT) tree geometry. We demonstrate that this geometry satisfies an emergent graph Einstein equation in a unique way that is consistent with the bulk effective matter action encoding the same correlation function as the tensor network, at least in the perturbative limit order by order away from the pure BT tree. Moreover, the (perturbative) definition of the graph curvature in the mathematics [Y. Lin and S.-T. Yau, Tohoku Math. J. 63, 605 (2011); Y. Ollivier, J. Funct. Anal. 256, 810 (2009)] and physics [S. S. Gubser et al., J. High Energy Phys. 06 (2017) 157] literature naturally emerges from the consistency requirements of the emergent Einstein equation. This could provide new insights into the understanding of gravitational dynamics potentially encoded in more general tensor networks.
\end{abstract}

DOI: 10.1103/PhysRevLett.127.221602

The AdS/CFT correspondence [1] provided deep insights of (quantum) gravity. One extremely important breakthrough inspired by the Ryu-Takayanagi entanglement formula [2] is the realization that (semiclassical) geometries as solutions of the gravity theory are basically geometrization of the entanglement structure of wave functions of the dual conformal field theory (CFT). (See for example Ref. [3] for a review and references therein.) Tensor networks (TN) widely used to construct many-body wave functions are also geometrizations of patterns of entanglement of many-body wave functions. This led to proposals that TNs similar to the multiscale entanglement renormalization ansatz capture the microscopic mechanism behind the AdS/CFT correspondence [4]. Toy models have been constructed [5,6] that recreate many aspects of the AdS/CFT correspondence, most notably the Ryu-Takayanagi formula and the error correcting property [7]. TN also provides deep insight in the surface-state correspondence [8], kinematic space [9], the complexity of the wave functions and their evolution [10-14], and the island formula that is probably the key to the black hole information paradox $[15,16]$. Nonetheless, reconstruction of gravitational dynamics using $\mathrm{TN}$ remains a tremendous challenge. There is some progress by considering relative entropies $[17,18]$ and also complexity

Published by the American Physical Society under the terms of the Creative Commons Attribution 4.0 International license. Further distribution of this work must maintain attribution to the author(s) and the published article's title, journal citation, and DOI. Funded by SCOAP ${ }^{3}$. optimization [12-14], although it is not completely clear how bulk matter or generic time dependence can be included. (Some progress was recently made in Ref. [19].)

In this paper, we present the emergence of a graph Einstein equation based on the proposed TN [20] of the $p$-adic AdS/CFT [21,22]. Our strategy is to parametrize the form of curvatures and bulk stress tensor using an ansatz that is based only on locality and symmetry. We will show that if an Einstein equation exists at all the self-consistency constraints are stringent enough to return a unique one from the TN. This is perhaps the first such quantitative demonstration involving both matter and time [23], and where both the CFT and the TN can be explicitly defined, taking advantage of the simplicity of $p$-adic CFT, where descendent operators do not feature in conformal blocks.

Lightning review of $\mathrm{p}$-adic CFTs. - A one-dimensional $p$-adic CFT lives in the $p$-adic number field $Q_{p}$ for any given prime number $p$, i.e., coordinates $x \in Q_{p} . p$-adic numbers $Q_{p}$ are field extensions of the rational numbers alternative to the reals $\mathbb{R}$. This can be readily generalized to an $n$-dimensional $p$-adic CFT by considering the field extension of $Q_{p}$ to $Q_{p^{n}}$ [21,22]. To avoid clutter we will take $n=1$ although all the expressions can be generalized for generic $n$, basically by replacing $p \rightarrow p^{n}$. A $p$-adic number $x$ can be uniquely expressed as an infinite series

$$
x=p^{v} \sum_{i=0} a_{i} p^{i} ; \quad v, a_{i} \in \mathbb{Z} ; \quad 0 \leq a_{i} \leq p-1 ; \quad a_{0} \neq 0 .
$$

The $p$-adic norm is defined as 


$$
|x|_{p}=p^{-v},
$$

which satisfies various axioms of norms [24]. Conformal symmetry is defined as the transformation

$x \rightarrow x^{\prime}=\frac{a x+b}{c x+d}, \quad a, b, c, d \in Q_{p}, \quad a d-b c \neq 0$.

It furnishes the matrix group $\operatorname{PGL}\left(2, Q_{p}\right)$, the direct analogue of $\operatorname{SL}(2, \mathbb{R})$ in $1 \mathrm{D}$ conformal transformation in real space-time. There are two pieces of algebraic data required to specify completely a $p$-adic CFT [25]: (i) the spectrum of (quasi) primary operators $\mathcal{O}_{a}$ with conformal dimensions $\Delta_{a}$, which transform under conformal symmetry as

$$
\mathcal{O}_{a}(x) \rightarrow \tilde{\mathcal{O}}_{a}\left(x^{\prime}\right)=\left|\frac{a d-b c}{(c x+d)^{2}}\right|_{p}^{-\Delta_{a}} \mathcal{O}_{a}(x)
$$

and (ii) operator product expansion (OPE) coefficients $C^{a b c}$ defined as [26]

$$
\mathcal{O}_{a}\left(x_{1}\right) \mathcal{O}_{b}\left(x_{2}\right)=\sum_{c} C^{a b c}\left|x_{1}-x_{2}\right|_{p}^{\Delta_{c}-\Delta_{a}-\Delta_{b}} \mathcal{O}_{c}\left(x_{2}\right) .
$$

The OPE coefficients define an associative operator fusion, i.e., $\sum_{c} C^{a b c} C^{c d e}=\sum_{c} C^{b d c} C^{c a e}$. There is a unique identity operator II so that $C^{1 a b}=C^{a 1 b}=\delta_{a b}$, and that there is a unique dual of $a$ which we denote as $a *$ satisfying $C^{a b 1}=\delta_{b a *}$. Assuming $a *=a$ and $C^{a b 1}=\delta_{a b}$, the edges need not be oriented, and the notations are less cluttered. This assumption will be taken in the rest of the paper, but it is readily generalized to cases where $a \neq a *$ [20].

TN on the Bruhat-Tits tree and the p-adic AdS/CFT.The partition function of a generic $p$-adic CFT can be constructed explicitly in the form of a TN covering the Bruhat-Tits tree [20], the latter of which is a discrete $p+1$ valent tree graph. The BT tree is the analog of the $\mathrm{AdS}_{2}$ space whose isometry is the corresponding conformal symmetry group. The TN covers the tree, such that at each vertex of the tree sits a $p+1$ index tensor $T^{a_{1} \cdots a_{p+1}}$. The tensor is given by a fusion tree of $p+1$ operators, expressed in terms of the OPE coefficient of the $p$-adic CFT [20].

$$
T^{a_{1} \cdots a_{p+1}}=\sum_{b_{1} \cdots b_{p-2}} C^{a_{1} a_{2} b_{1}} C^{b_{1} a_{3} b_{2}} \cdots C^{b_{p-2} a_{p} a_{p+1}} .
$$

In the special case where $p=2, T^{a b c}=C^{a b c}$. Two tensors at two vertices connected by an edge are contracted with the edge index weighted by $p^{-\Delta_{a}}$, where $\Delta_{a}$ is the conformal dimension of the corresponding primary labeled $a$. The asymptotic boundary of the tree is $Q_{p}$, analogous to the real line being the asymptotic boundary of the $\mathrm{AdS}_{2}$ space. The partition function is defined by setting appropriate boundary conditions at the cut-off surface which is then taken to approach the asymptotic boundary. Specifically, the dangling legs at the cut-off surface $\Lambda$ are projected along the vector $\left|V_{f}\right\rangle \equiv \sum_{a} \delta_{1}^{a}|a\rangle=|1\rangle$ [20]. To compute correlation functions, one inserts operator $\mathcal{O}_{a}\left(x_{b}\right)$ by projecting the boundary leg at $x_{b}$ along $|a\rangle$ instead. The $\mathrm{TN}$ reproduces the correct correlation function in the form of Witten-like diagrams [20]. The boundary insertions of $\mathcal{O}_{a}\left(x_{b}\right)$ source these $p^{-\Delta_{a}}$ weighted paths in the TN, which coincides with "bulk-boundary propagators" $G_{a}\left(x_{b}, v_{i}\right)$ on the tree, until they meet at some vertices $v_{i}$ in the bulk. These bulk-boundary propagators are solutions of the graph Klein-Gordon equation

$\left(\square_{v}+m_{a}^{2}\right) G_{a}(u, v)=\delta_{u, v}, G_{a}(u, v)=\zeta_{p}\left(2 \Delta_{a}\right) \frac{p^{-\Delta_{a} d(u, v)}}{p^{\Delta_{a}}}$,

$$
\begin{aligned}
& m_{a}^{2}=-\frac{1}{\zeta_{p}\left(\Delta_{a}-1\right) \zeta_{p}(-\Delta)}, \quad \zeta_{p}(s) \equiv \frac{1}{1-p^{-s}} \\
& \square_{u} \phi(u) \equiv \sum_{\langle u v\rangle, v \sim u}[\phi(u)-\phi(v)], \quad u \sim v \equiv \text { nearest neighbor. }
\end{aligned}
$$

Here $\langle u v\rangle$ denotes an edge ending at vertices $u, v$, and $d(u, v)$ is the distance between $u, v$. Here, every link has unit length $d_{e}=1$. Operators labeled $a, b, \ldots$ traversing different paths can meet at a vertex if they can fuse to the identity operator. The emergence of Witten-diagrams suggests that the TN is recreating the $p$-adic AdS/CFT correspondence proposed in $[21,22]$, connecting a $p$-adic CFT and a dual bulk theory containing some bulk fields $\phi^{a}$ living on the BT tree in 1-1 correspondence with the primary operators. In fact, we can define bulk field $\phi^{a}(x)$ insertion by fusing an extra $a$ leg to the bulk vertex $x$. The bulk correlation functions $\left\langle\phi^{a}(x) \phi^{b}(y) \cdots\right\rangle$ are thus defined as evaluation of the tensor network with the extra legs inserted at the appropriate vertices (see Fig. 1).

One can readily show that these results are consistent with an emergent bulk matter field theory living on the BT tree [20,21], with action in the large mass limit [27]

$$
\begin{aligned}
S_{m}= & \sum_{\langle x y\rangle} \frac{1}{2}\left\{\sum_{a}\left[\phi^{a}(x)-\phi^{a}(y)\right]^{2}\right. \\
& \left.+\frac{m_{a}^{2}}{p+1}\left[\phi^{a}(x)^{2}+\phi^{a}(y)^{2}\right]\right\} \\
& +\sum_{a, b, c} \frac{\tilde{C}^{a b c}}{3 !(p+1)}\left[\phi^{a}(x) \phi^{b}(x) \phi^{c}(x)\right. \\
& \left.+\phi^{a}(y) \phi^{b}(y) \phi^{c}(y)\right]+\cdots \\
\tilde{C}^{a b c} \equiv & C^{a b c} \sqrt{\frac{p^{\Delta_{a}+\Delta_{b}+\Delta_{c}}}{\zeta_{p}\left(2 \Delta_{a}\right) \zeta_{p}\left(2 \Delta_{b}\right) \zeta_{p}\left(2 \Delta_{c}\right)}}
\end{aligned}
$$




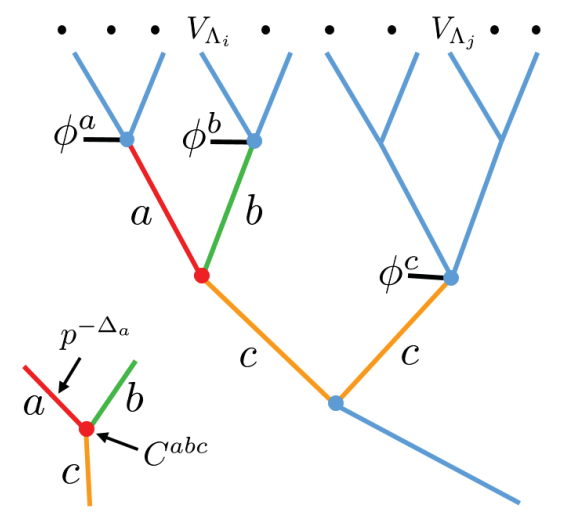

FIG. 1. The tensor network representation of a $p=2$-adic CFT. The diagram depicts three bulk operator insertion. Bulk insertions pushed to the asymptotic boundary asymptotes to boundary insertions. The boundary condition $V_{\Lambda_{i}}$, defined in (11), are chosen to be the fixed point tensor $V_{f}^{a}=\delta_{1}^{a}$. Each vertex tensor is $C^{a b c}$ and each edge of the tensor with index $a$ is weighted by $p^{-\Delta_{a}}$.

where $\cdots$ corresponds to higher point interaction terms. The effective action truncates at the $\phi^{p+1}$ level, which reduces to cubic vertices at $p=2$ [20]. We deliberately present the action as a sum over edges anticipating coupling the theory to a dynamical metric shortly.

Distances and curvatures in a tensor network.-The tensor network constructed appears to be describing a pure BT space (analog of pure AdS) when the boundary edges are projected to the identity vector apart from locations where operators are inserted. As in the usual story of AdS/CFT, a most natural way to deform the background geometry is to change the boundary conditions which would drive an renormalization group flow in the CFT. Specifically, each boundary link $i$ at the cutoff surface $\Lambda$ is projected to a generic vector $\left|V_{\Lambda_{i}}\right\rangle$ [20]. Where there is translation invariance, we can take $\left|V_{\Lambda_{i}}\right\rangle=\left|V_{\Lambda}\right\rangle$ for all boundary legs $i$. Explicitly the vector $\left|V_{\Lambda}\right\rangle$ is parametrized as

$$
\left|V_{\Lambda}\right\rangle=\sum_{a} V_{\Lambda}^{a}|a\rangle
$$

When the vectors $\left|V_{\Lambda}\right\rangle$ are contracted with the $p$ dangling legs of a tensor $T^{a_{1} \cdots a_{p+1}}$ at the cut-off surface, it would generate a new vector $\left|V_{\Lambda-1}\right\rangle$

$$
\begin{aligned}
\left|V_{\Lambda-1}\right\rangle & \equiv \sum_{a} V_{\Lambda-1}^{a}|a\rangle \\
& =\sum_{a_{1} \cdots a_{p+1}} V_{\Lambda}^{a_{1}} p^{-\Delta_{a_{1}}} \cdots V_{\Lambda}^{a_{p}} p^{-\Delta_{a_{p}}} T^{a_{1} \cdots a_{p} a_{p+1}}\left|a_{p+1}\right\rangle
\end{aligned}
$$

which is fed into the next layer of tensors recursively. The flow of these vectors suggests that the geometry described by the tensor network is deformed from the pure BT background. We note that the original boundary condition describing the undeformed CFT partition function

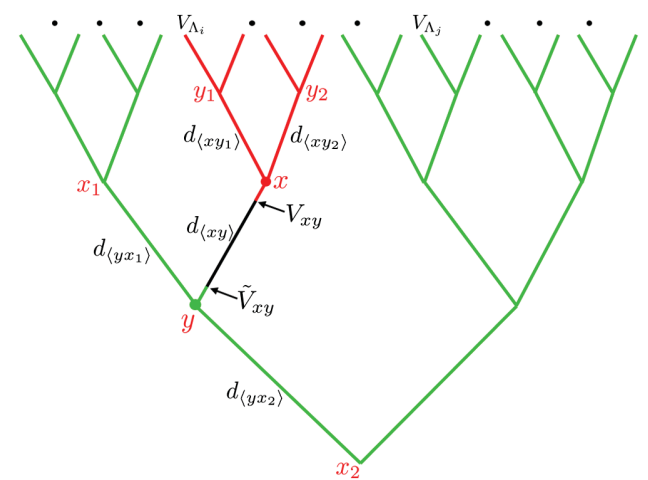

FIG. 2. Vector $V_{x y}^{a}$ and $\tilde{V}_{x y}^{a}$ following from the contraction of tensors above (colored red) and below (colored green) the edge $\langle x y\rangle$ respectively. Boundary conditions $V_{\Lambda_{i}}$ differing from the fixed point vector drives an renormalization group flow. The curvature of the patch centered at $x$ depends on the edge lengths $d_{\left\langle x y_{i}\right\rangle}$ symmetrically.

$$
V_{f}^{a}=\delta_{1}^{a}
$$

is indeed a fixed point vector under this flow, which recovers the original pure BT space supposedly dual to the undeformed $p$-adic CFT partition function. In this case, all the edges contribute equally to the partition function, and we can assign unit length to every edge, i.e., $d_{e}=1$.

When we depart from pure BT background, one needs to assign a general length $d_{e}$ to each edge on the graph. We note that there are two characteristic vectors at an edge $e=\langle x y\rangle$ bounded by vertices $x$ and $y$. One is $\left|V_{x y}\right\rangle$ introduced above, corresponding to repeating the flow described in Eq. (12), contracting all the tensors from the cut-off surface all the way down to the vertex $x$. The other vector $\left|\tilde{V}_{x y}\right\rangle$ follows from analogously contracting all the tensors below the vertex $y$. This misleading notion of being "above" or "below" is illustrated in Fig. 2.

The distinction between $\left|V_{x y}\right\rangle$ and $\left|\tilde{V}_{x y}\right\rangle$ comes from boundary conditions very far away, and so we expect the edge length $d_{\langle x y\rangle}$ to depend on them symmetrically. It is also natural to expect that $\left|V_{x y}\right\rangle$ and $\left|\tilde{V}_{x y}\right\rangle$ fully determine $d_{\langle x y\rangle}$. To have better analytic control, we consider perturbing around the undeformed CFT by perturbing the boundary conditions $\left|V_{\Lambda}\right\rangle$ around the fixed point tensor:

$$
V_{\Lambda_{i}}^{a}=\delta_{1}^{a}+\lambda v_{\Lambda_{i}}^{a},
$$

where $\lambda \ll 1$, but $v_{\Lambda_{i}}^{a}$ is completely arbitrary. The boundary conditions are not assumed to respect any translation symmetries. In the perturbative limit, the flowed vector in the interior would admit the general expansion

$$
\begin{array}{ll}
V_{x y}^{a}=\delta_{1}^{a}+\omega_{x y}^{a}, & \omega_{x y}^{a} \equiv \lambda_{x y}^{(1) a}+\lambda_{x y}^{(2) a}+\cdots, \\
\tilde{V}_{x y}^{a}=\delta_{1}^{a}+\tilde{\omega}_{x y}^{a}, & \tilde{\omega}_{x y}^{a} \equiv \tilde{\lambda}_{x y}^{(1) a}+\tilde{\lambda}_{x y}^{(2) a}+\cdots,
\end{array}
$$


where $\lambda^{(n)}, \tilde{\lambda}^{(n)}$ are of order $\lambda^{n}$. The edge length $d_{e=\langle x y\rangle}\left(V_{e}^{a}, \tilde{V}_{e}^{a}\right)$ would also admit an expansion about the pure BT space as $d_{e}=1+j_{e}$, where

$$
\begin{aligned}
j_{e}= & A^{a}\left(\omega_{e}^{a}+\tilde{\omega}_{e}^{a}\right)+B^{a b}\left(\omega_{e}^{a} \omega_{e}^{b}+\tilde{\omega}_{e}^{a} \tilde{\omega}_{e}^{b}\right)+C^{a b} \omega_{e}^{a} \tilde{\omega}_{e}^{b} \\
& +D^{a b c}\left(\omega_{e}^{a} \omega_{e}^{b} \omega_{e}^{c}+\tilde{\omega}_{e}^{a} \tilde{\omega}_{e}^{b} \tilde{\omega}_{e}^{c}\right) \\
& +E^{a b c}\left(\omega_{e}^{a} \omega_{e}^{b} \tilde{\omega}_{e}^{c}+\tilde{\omega}_{e}^{a} \tilde{\omega}_{e}^{b} \omega_{e}^{c}\right)+\mathcal{O}\left(\omega^{4}\right),
\end{aligned}
$$

for some constants $A^{a}, B^{a b}, C^{a b} \ldots$. Having assigned edge lengths, one can compute the curvature of this graph. There are various proposals, e.g., Refs. [28-30]. Consider the curvature $R_{x}$ of a patch surrounding a vertex $x$. The graph curvature should be a symmetric function of lengths $d_{\left\langle x y_{i=1, \ldots p+1}\right\rangle}$ of the $p+1$ edges connected to $x$. Generally therefore, in the small $j_{e}$ limit we expect

$$
\begin{aligned}
R_{x}= & a_{0}+a_{1} \sum_{i} j_{x y_{i}}+b \sum_{i} j_{x y_{i}}^{2}+c \sum_{i \neq k} j_{x y_{i}} j_{x y_{k}} \\
& +\mathcal{O}\left(j^{3}\right),
\end{aligned}
$$

again for constants $a_{0,1}, b, c \cdots$.

Emergent action and Einstein equation. - In the above, we have defined distances and curvatures which are, up to some undetermined coefficients, determined by the TN. On the other hand, the TN encodes a bulk scalar field theory where the expectation values of the fields $\phi^{a}(x)$ can be readily computed. We would therefore like to inquire if the geometry and the expectation values of $\phi^{a}(x)$, both read off from the $\mathrm{TN}$, can be related by some graph Einstein equation.

To look for such a relation, we need an emergent covariant effective action that describes the bulk theory, and obtains the equations of motion following from it, before we could even check if such an equation is satisfied by the TN. The matter part of the covariant action should reduce to Eq. (10) in the pure BT limit to be consistent with the correlation functions. We need to upgrade Eq. (10) by coupling it to the background geometry via the edge lengths $d_{e}$. This is attempted in Ref. [30], although it has made a choice of treating the mass term as a local term blind to $d_{e}$. This is unlike in continuous field theories where every term contains at least the volume form and are thus always sensitive to the metric. Rather than making such choices, we write down a more general ansatz that mimics the continuous covariant action more closely,

$$
\begin{aligned}
S_{m}^{\mathrm{cov}}= & S_{2}^{\mathrm{cov}}+S_{3}^{\mathrm{cov}}+\cdots \\
S_{2}^{\mathrm{cov}}= & \sum_{\langle x y\rangle} d_{\langle x y\rangle}^{k}\left(\phi_{x}^{a}-\phi_{y}^{a}\right)^{2}+\sum_{\langle x y\rangle} \frac{d_{\langle x y\rangle}}{p+1} m_{a}^{2}\left[\left(\phi_{x}^{a}\right)^{2}+\left(\phi_{y}^{a}\right)^{2}\right] \\
S_{3}^{\mathrm{cov}}= & \sum_{\langle x y\rangle}\left(h\left(d_{\langle x y\rangle}\right) H^{a b c}\left(\phi_{x}^{a} \phi_{x}^{b} \phi_{x}^{c}+\phi_{y}^{a} \phi_{y}^{b} \phi_{y}^{c}\right)\right. \\
& \left.+r\left(d_{\langle x y\rangle}\right) R^{a b c}\left(\phi_{x}^{a} \phi_{x}^{b} \phi_{y}^{c}+\phi_{y}^{a} \phi_{y}^{b} \phi_{x}^{c}\right)\right),
\end{aligned}
$$

where $k$ is a constant to be determined. For generality we considered more general cubic interactions other than the exactly local term with coupling $H^{a b c}$, and allowed also for nearest neighbor interaction with coupling $R^{a b c}$. The functions $r\left(d_{e}\right)$ and $h\left(d_{e}\right)$ should be regular in the pure BT background. Therefore in the perturbative limit they can be expanded as

$$
\begin{gathered}
h\left(d_{x y}\right)=h_{0}+h_{1} j_{x y}+h_{2} j_{x y}^{2}+\ldots, \\
r\left(d_{x y}\right)=r_{0}+r_{1} j_{x y}+r_{2} j_{x y}^{2}+\ldots
\end{gathered}
$$

We introduce the graph Einstein Hilbert action making use of the graph curvature introduced in Eq. (18).

$$
S_{\mathrm{EH}}=\sum_{x} R_{x}\left(d_{x y_{1}}, d_{x y_{2}}, \ldots, d_{x y_{p+1}}\right)+\sum_{\langle x y\rangle} d_{x y} \Lambda,
$$

where we introduced also the cosmological constant term analogous to $\int d^{d} x \sqrt{g} \Lambda$. The total test effective action is thus $S_{\text {tot }}=S_{\mathrm{EH}}+S_{m}^{\text {cov }}$. Now we are ready to vary these actions with respect to each edge length $d_{\langle x y\rangle}$ to obtain the graph Einstein equation. Varying $S_{\mathrm{EH}}$ we obtain the graph Einstein tensor $G$ :

$$
\begin{aligned}
G_{x y} \equiv & \frac{\delta S_{\mathrm{EH}}}{\delta d_{x y}} \\
= & \Lambda+2 a_{1}+4 b j_{x y}+c\left(\sum_{\substack{i \\
\left(y_{i} \neq y\right)}} j_{x y_{i}}+\sum_{\substack{i \\
\left(x_{i} \neq x\right)}} j_{x_{i} y}\right) \\
& +\mathcal{O}\left(j^{2}\right) .
\end{aligned}
$$

Varying $S_{m}^{\text {cov }}$ we obtain

$$
\begin{aligned}
T_{x y} \equiv & \frac{\delta S_{m}^{\mathrm{cov}}}{\delta d_{x y}} \\
= & \frac{k}{2}\left(\phi_{x}^{a}-\phi_{y}^{a}\right)^{2}+\frac{m_{a}^{2}\left[\left(\phi_{x}^{a}\right)^{2}+\left(\phi_{y}^{a}\right)^{2}\right]}{2(p+1)} \\
& +\left[h_{1} H^{a b c}\left(\phi_{x}^{a} \phi_{x}^{b} \phi_{x}^{c}+\phi_{y}^{a} \phi_{y}^{b} \phi_{y}^{c}\right)+r_{1} R^{a b c}\left(\phi_{x}^{a} \phi_{x}^{b} \phi_{y}^{c}\right.\right. \\
& \left.\left.+\phi_{y}^{a} \phi_{y}^{b} \phi_{x}^{c}\right)\right]+\cdots .
\end{aligned}
$$

We would like to substitute the expectation values of $\phi^{a}(x)$ into $T$. We provide detailed expressions of $\phi^{a}(x)$ in the Supplemental Material [31]. Clearly $\phi^{a}(x) \sim \lambda$, and the omitted terms in Eq. (24) are thus of order $\lambda^{4}$ and beyond. The graph Einstein equation is thus given by

$$
G_{x y}+T_{x y}=0 .
$$

We substitute the geometrical data and expectation value of the bulk stress tensor determined by the TN into Eq. (25). Requiring that Eq. (25) is satisfied order by order in $\lambda$ turns 
into constraints of the undetermined parameters we have introduced. These constraints turn out to be very powerful because $G_{x y}$ and $T_{x y}$ are nontrivial functions of $\lambda_{x y_{i}}^{(n) a}, \tilde{\lambda}_{x y_{i}}^{(n) a}$ and $\lambda_{y x_{i}}^{(n) a}, \tilde{\lambda}_{y x_{i}}^{(n) a}$. These edges $\left\langle x y_{i}\right\rangle,\left\langle y x_{i}\right\rangle$ are marked in Fig. 2 for a given pair of connected vertices $x, y$. These variables $\lambda_{x y_{i}}^{(n) a}, \tilde{\lambda}_{y x_{i}}^{(n) a}$ are virtually independent because they are distinct functions of generic boundary conditions arbitrarily far away. Therefore, we can isolate the coefficient of each of these independent monomials of $\lambda_{x y_{i}}^{(n) a}, \tilde{\lambda}_{y x_{i}}^{(n) a}$, and require that it vanishes separately. Up to some overall normalization, it fixes a unique perturbative dependence of the graph curvature on the edges, the edge lengths, and coupling of matter to the edge lengths such that Eq. (25) can in fact be satisfied. Solving constraints up to order $\lambda^{2}$, we have

$$
\begin{aligned}
\frac{2 b}{c}=-p, \quad A^{a} & =0, \quad \Lambda+2 a_{1}=0, \\
k & =1 .
\end{aligned}
$$

Substituting into $G_{x y}$ the linear $j$ term is equivalent to the perturbative graph curvature defined in the literature, specifically the form emerging in Ref. [30], up to an overall normalization $-c$. We note that the relation in Eq. (8) has been used when solving the constraints. $B^{a b}$ and $C^{a b}$ introduced in Eq. (17) are in turn determined by $b, c$, and $\Delta_{a}$. Constraints at order $\lambda^{3}$ again lead to unique expressions for the matter couplings $H^{a b c}, R^{a b c}$ and edge length expansion coefficients $D^{a b c}$, $E^{a b c}$ up to an overall undetermined normalization. More detailed expressions and explanations are relegated to the Supplemental Material [31]. We are interested in the limit $m_{a}^{2} \rightarrow \infty \Rightarrow \Delta_{a} \rightarrow \infty$. This is when the bulk effective action $S_{m}$ exactly reproduces $\phi^{a}(x)$ correlation functions of the TN. In this limit, satisfying only the local term survives with an overall undetermined normalization,

$$
\lim _{\Delta_{a} \rightarrow \infty, d_{e} \rightarrow 1} S_{3}^{\mathrm{cov}}=-\frac{h_{0}}{4 h_{1}} \sum_{\langle x y\rangle} \frac{\tilde{C}^{a b c}}{1+p} \phi_{x}^{a} \phi_{x}^{b} \phi_{x}^{c},
$$

but otherwise in exact agreement with the effective action [Eq. (10)].

Summary and discussion.-In this paper, we demonstrated that there is, up to some overall normalization, a unique way of assigning lengths to the $p$-adic TN so that the geometry read off from the TN satisfies a graph Einstein equation that is consistent with the bulk effective action that reproduces bulk correlation functions encoded by the TN, in the perturbative limit away from pure BT geometry. We have made minimal assumptions other than locality in our ansatz for the action and also the dependence of edge lengths on the TN data. We note that in retrospect, the edge distance $d_{\langle x y\rangle}=1+j_{x y}$ defined in Eq. (17) can be written as

$$
d_{\langle x y\rangle}=1-\left\langle u_{x} \mid u_{y}\right\rangle
$$

whose complete expression is in the Supplemental Material [31]. In the limit $\Delta_{a} \rightarrow \infty$,

$$
\left|u_{x}\right\rangle=\frac{1}{\sqrt{2(p+1)}} \sum_{a} \tilde{\phi}_{x}^{a}|a\rangle .
$$

Up to the overall factor in front, this is simply the state corresponding to one dangling leg inserted at a bulk vertex $x$ in the TN. Indeed, $d_{\langle x y\rangle}$ ends up being a Fisher information metric between these vertex states. This is a rare quantitative demonstration of an emergent Einstein equation from a TN that couples to matter, albeit in a simplified setting of $p$-adic CFTs. There are more patterns in the emergent graph Einstein equation that we will report in a forthcoming accompanying paper. We believe this provides further evidence of geometry being molded by the correlation of matter, supporting the $\mathrm{TN}$ as the microscopic mechanism behind the AdS/CFT correspondence. We believe some ideas and methodology discussed here should admit generalization to TN describing more realistic CFTs.

L. Y.H. acknowledges the support of NSFC (Grants No. 11922502, No. 11875111) and the Shanghai Municipal Science and Technology Major Project (Shanghai Grant No. 2019SHZDZX01). This research was supported in part by the National Science Foundation under Grant No. NSF PHY-1748958. Part of this work was instigated in KITP during the program qgravity20. L. C. acknowledges support of NSFC (Grant No. 12047515). We thank Bartek Czech, Ce Shen Gabriel Wong, Qifeng Wu, and Zhengcheng $\mathrm{Gu}$ for useful discussions and comments. We thank Si-nong Liu and Jiaqi Lou for collaboration on related projects.

* Corresponding author. lyhung@fudan.edu.cn Corresponding author. linchen91@pku.edu.cn

[1] J. M. Maldacena, Int. J. Theor. Phys. 38, 1113 (1999).

[2] S. Ryu and T. Takayanagi, Phys. Rev. Lett. 96, 181602 (2006).

[3] M. Van Raamsdonk, in Theoretical Advanced Study Institute in Elementary Particle Physics: New Frontiers in Fields and Strings (World Scientific Publishing Company, Singapore, 2017), pp. 297-351.

[4] B. Swingle, Phys. Rev. D 86, 065007 (2012).

[5] F. Pastawski, B. Yoshida, D. Harlow, and J. Preskill, J. High Energy Phys. 06 (2015) 149.

[6] P. Hayden, S. Nezami, X.-L. Qi, N. Thomas, M. Walter, and Z. Yang, J. High Energy Phys. 11 (2016) 009. 
[7] A. Almheiri, X. Dong, and D. Harlow, J. High Energy Phys. 04 (2015) 163.

[8] M. Miyaji, T. Numasawa, N. Shiba, T. Takayanagi, and K. Watanabe, Phys. Rev. Lett. 115, 171602 (2015).

[9] B. Czech, L. Lamprou, S. McCandlish, and J. Sully, J. High Energy Phys. 07 (2016) 100.

[10] L. Susskind, Fortschr. Phys. 64, 24 (2016); 64, 44(A) (2016).

[11] A. R. Brown, D. A. Roberts, L. Susskind, B. Swingle, and Y. Zhao, Phys. Rev. D 93, 086006 (2016).

[12] P. Caputa, N. Kundu, M. Miyaji, T. Takayanagi, and K. Watanabe, Phys. Rev. Lett. 119, 071602 (2017).

[13] P. Caputa, N. Kundu, M. Miyaji, T. Takayanagi, and K. Watanabe, J. High Energy Phys. 11 (2017) 097.

[14] B. Czech, Phys. Rev. Lett. 120, 031601 (2018).

[15] G. Penington, J. High Energy Phys. 09 (2020) 002.

[16] A. Almheiri, R. Mahajan, J. Maldacena, and Y. Zhao, J. High Energy Phys. 03 (2020) 149.

[17] T. Faulkner, M. Guica, T. Hartman, R. C. Myers, and M. Van Raamsdonk, J. High Energy Phys. 03 (2014) 051.

[18] T. Faulkner, J. High Energy Phys. 05 (2015) 033.

[19] J. Boruch, P. Caputa, and T. Takayanagi, Phys. Rev. D 103, 046017 (2021).

[20] L.-Y. Hung, W. Li, and C. M. Melby-Thompson, J. High Energy Phys. 04 (2019) 170.
[21] S. S. Gubser, J. Knaute, S. Parikh, A. Samberg, and P. Witaszczyk, Commun. Math. Phys. 352, 1019 (2017).

[22] M. Heydeman, M. Marcolli, I. Saberi, and B. Stoica, Adv. Theor. Math. Phys. 22, 93 (2018).

[23] Here time corresponds to an extra coordinate. p-adic CFT is basically a Euclidean theory with no distinction of space and time.

[24] A. Ostrowski, Acta arithmetica 41, 271 (1916).

[25] E. Melzer, Int. J. Mod. Phys. A 04, 4877 (1989).

[26] Note that this expression is exact since $p$-adic CFT is known to have no descendents.

[27] The interaction vertex is essentially fixed at the meeting point of geodesics on the tree rather than summed over [20]. Therefore it agrees with the semi-classical limit of a massive field.

[28] Y. Lin and S.-T. Yau, Tohoku Math. J. 63, 605 (2011).

[29] Y. Ollivier, J. Funct. Anal. 256, 810 (2009).

[30] S. S. Gubser, M. Heydeman, C. Jepsen, M. Marcolli, S. Parikh, I. Saberi, B. Stoica, and B. Trundy, J. High Energy Phys. 06 (2017) 157.

[31] See Supplemental Material at http://link.aps.org/supplemental/ 10.1103/PhysRevLett.127.221602 for the details of the perturbative expansion away from the pure BT tree limit. 